\title{
Psychology: The study of green cheese
}

\author{
KENNETH R. BURSTEIN \\ Simon Fraser University, Burnaby, British Columbia V5A 1S6, Canada
}

\begin{abstract}
Three distinctive facets of classical behaviorism are critically examined. The first facet, methodological behaviorism, constitutes a straightforward commitment to empiricism and is, therefore, not controversial. However, the second facet, theoretical behaviorism, is found to be extreme and anachronistic. The final facet, so-called substantial behaviorism, involving, as it does, a change in the subject matter of psychology, is shown to be seriously in error and inconsistent with modern practices in the "hard" sciences.
\end{abstract}

"As a result of this major assumption that there is such a thing as consciousness and that we can analyze it by introspection, we find as many analyses as there are individual psychologists" (Watson, 1925, pp. 5-6).

With this summary of the frustration generated by the structuralist approach, John B. Watson, among others, called for the abandonment of introspection, as the method, and of mind, as the subject matter, of psychology, and "behaviorism" took root in the American psychological scene. While this is, of course, fairly common knowledge, what is sometimes apparently overlooked is that behaviorism, as introduced by Watson, had at least three different facets, each with important ramifications of its own.

First, it called for a change in methodology (methodological behaviorism). Second, it presented a particular theoretical overview (theoretical behaviorism). Third, it called for a change in the subject matter of psychology (substantial behaviorism). These three different aspects of behaviorism merit close examination.

With respect to methodological behaviorism, there would seem to be little about which one could argue. While one occasionally meets a psychologist who proudly proclaims "I am a behaviorist" and, perhaps just as frequently, encounters someone who sneeringly accuses others of being behaviorists, if the term is used in its methodological sense, there simply is no issue. One is tempted to ask "Behaviorist as opposed to what?" Behaviorism, in its methodological sense, is equivalent to empiricism, and it makes as much sense for a chemist or a biologist or a physicist to cry that he is, or is not, an empiricist. Time and history have demonstrated that, although alternatives to empiricism exist, none are satisfactory. Thus, while it is doubtful that anyone would object to knowledge or "answers" obtained from a Ouija board, or mystical experiences, or plain old "common sense," such sources of information have been found to be quite unsatisfactory.

Common sense tells us that the earth is flat, does not move, is the center of the universe, and is closer to the sun in the summer. The empirical approach provides a protective mechanism against these subjectively verifiable conclusions.
In its first and methodological sense, therefore, the term "behaviorist" is relatively meaningless, and some consideration should be given to abandoning its use or to replacing it with the term "empiricist." In this way, some might come to replace an emotional response to the word "behaviorism" with a tenable and clearly stated alternative methodological position.

With respect to theoretical behaviorism, there simply are not many, if any, Watsonian behaviorists around. Clearly, those who cry that they are or are not behaviorists are not referring to behaviorism in its theoretical sense. Watsonian behaviorism is far too extreme with respect to the role of covert events, of conditioned reflexes, and of heredity to be adopted in numbers large enough to justify a schism of the magnitude implied by the frequent usage of the term "behaviorist." Thus, the term "behaviorist," in its theoretical sense, should be used approximately as frequently as one uses the term "phlogistonist," or "caloricist," or "etherist."

We now come to the third aspect of behaviorism, as introduced by Watson (1925): the change of the subject matter of psychology from mind or consciousness to behavior. Although this is not the issue that typically leads psychologists to lift their "behaviorist" or "antibehaviorist" placards, it perhaps should be, for this change in the subject matter of the field, introduced by Watson and uncritically accepted by the overwhelming majority of those in the discipline, has possibly done more to hinder progress and understanding than has any other idea or notion in the history of psychology.

The pernicious effect of this aspect of behaviorism is largely the result of a failure to discriminate between method and subject matter. It is as if this change in subject matter were required by the adoption of the empirical approach. Nothing could be further from the truth. Indeed, psychology is the only empirical science that has attempted to formally limit itself to the study of the directly observable events required by the empirical method, and the only one that has equated its subject matter with these overt events. Clearly, chemistry, physics, and astronomy are as hardnosed, as empirical, as scientific, and as "behavioristic" as even the most ardent Watsonian behaviorist would 
wish. Yet, these disciplines did not change their subject matter when they broke away from philosophical traditions and adopted methodological empiricism.

Methodological behaviorism was a giant step forward, a step that has been taken by every branch of inquiry in which there has been any appreciable progress. It asked quite simply that empirical support be provided for statements or conclusions before they were regarded as factual.

Substantial behaviorism was a giant step backward, for it essentially precluded, in the strict formal sense, the use of inferential statements and required that the subject matter be directly observable. Adoption of this view by American psychologists was essentially an admission that certain phenomena and processes, not directly observable, were, in principle, inaccessible to scientific inquiry and should, therefore, be formally banished by limiting the subject matter of psychology to observable behavior.

This may not be as obvious as it should be because, since the formal change of subject matter from mind or consciousness to behavior, inferential statements have, in various forms, gradually reappeared in increasing numbers in the literature. Some of the previously outlawed "mental" states reappeared with newly found names implying some overtly observable characteristic (e.g., affective response, perceptual response). Others appeared by redefining behavior in such a way that any energy change, be it molar or molecular, overt or covert, was admissible (e.g., fractional anticipatory goal response). Indeed, the pervasive presence of intervening variables and hypothetical constructs in contemporary psychological theories should constitute prima facie evidence that the subject matter of psychology is not observable behavior.

However, the real point is not that we should broaden the definition of behavior formally so that there is some agreement between what we study and what we say is the subject matter of the field. The real necessity is that we acknowledge the true subject matter of the field so that there can be a realistic appraisal of where progress is being made and where we are at an impasse. Such an objective appraisal is impossible as long as we legislate out of scientific domain questions that are not, at present, easily subjected to empirical inquiry.

Whether the word "mind" was spawned of ignorance and superstition or was first uttered by some addleheaded prebehavioristic primate, it fills a descriptive vacuum. It is a reminder of the kinds of questions people associate with the field of psychology. Clearly, some of those questions may be amenable to the empirical method and some may not. But the task of determining which, if any, of these questions are valid is not made easier by avoiding words that might remind us of the task. We have ceased raising our arms to etch apologetic quotation marks when we say "fear," although we still raise them for "disappointment," "relief," and "hope," because the GSR has legitimized fear as empirically acceptable subject matter, but we have nothing corresponding to the GSR to reflect hope, relief, and disappointment. Moreover, we are not even searching for such measures in order to determine whether hope, relief, and disappointment can be as valuable as variables as fear has been. What the apologetic quotation marks reflect are the perceived boundaries of the subject matter of the field. Similarly, rapid eye movements have legitimized dreams as proper subject matter.

It is better to have a multitude of phlogistons, calorics, and ethers and to search for empirical tests of these concepts than to apologize for them and then to legitimize them when someone stumbles upon or is handed a correlated observable event. Somehow psychologists are so defensive about their scientific training that they adopt poses and positions unbecoming a scientist. There are few physicists who apologetically raise their arms when speaking of "love," "charm," or "quarks." Indeed, the choice of names for these variables may involve a lesson about apologies and about proper subject matter that should be absorbed by psychologists. In a word then, the subject matter of psychology has not changed over the millenia: It is still the study of mind or consciousness-whatever that means. It is the task of the psychologist to find out what these crudely descriptive terms mean, or do not mean.

There are other terms, also having "mental" connotations, the avoidance of which should also be closely examined. A good many of us go into an introductory psychology or introductory learning class and ask, "What are the causes of a rat showing a tendency to run faster and faster to a goalbox in which food is consistently placed?" If we are lucky, some unsuspecting student replies, "The rat knows where the food is and he wants the food." We can then flaunt our level of sophistication before the class by tearing this "explanation" apart and, at the same time, informing the students of the cardinal sins sometimes committed by the inspired but unenlightened layman. First of all, we let the students know that attributing human traits to subhuman species is anthropomorphizing and is not to be tolerated, since there is no empirical basis for the attribution of human characteristics to animals. Second, we inform the students that, in explaining the rat's behavior in terms of an event that follows that behavior, we are guilty of a teleological explanation in that we are attributing purpose to the behavior. We wisely note that one cannot explain A in terms of B, if B follows $A$, and point out, as an example, that the eye does not grow or develop in order to see, or perhaps that sexual behaviors are not explained in terms of subsequent births.

With respect to the first sin, just as there is nothing wrong with a Ouija board, or messages felt as Morse code-like pulses in the medulla, as a source of hypotheses, there is nothing terrible about a hypothesis generated by anthropomorphizing. One cannot present such 
hypotheses as explanations unless they are accompanied by the kind of support demanded by the empirical method, but one cannot present any hypotheses, as explanations, without similar support.

In addition, one might consider the possibility that hypotheses generated by anthropomorphism have a higher probability of being correct than those obtained from a Ouija board or through revelation, in that the nervous systems of many animals are very similar to the human nervous system, which is apparently the reason that squid axons have been studied by people interested in human neural transmission, that Ham, a primate, was sent up in an earth-orbiting satellite, and that medical students spend many hours studying frogs, rats, and dogs. While it does not seem fruitful to assume that an isomorphic relationship between structure and function exists independent of phylogenetic niche, it does not seem reasonable, either, to assume that no relationship exists. If the human nervous system generates experiences reported as "hope," "fear," "relief," and "disappointment," it seems possible, logically at least, that other subhuman, but similar, nervous systems may also generate experiences that are roughly equivalent and have relatively similar influences upon overt behavior. Whether this question is answerable, at the present time, is of little consequence with respect to the legitimacy of asking the question. In any event, the sin of anthropomorphizing clearly is not in attributing human traits to subhuman species. We unquestioningly attribute a myriad of human traits to other species. The "sin" arises because we attribute traits or characteristics without providing empirical support. But the attribution of any traits, be they human or not, would be unacceptable if unaccompanied by empirical support. The wide usage and uncritical acceptance of the term "anthropomorphism," in a perjorative sense, almost implies that subhuman species cannot possibly possess traits or experiences similar to those of humans, when all that one can legitimately say is that it is difficult to demonstrate the existence of these human-like traits or experiences. The difficulty of demonstrating traits and experiences in subhuman species has little, if anything, to do with whether similar traits or experiences are possessed by humans.

Perhaps the point is best made by illustration. Consider a situation in which intelligent, articulate, adult human beings are run through a giant maze-like structure in which the "goalbox" contains stocks, bonds, escargots, and other highly prized reinforcers. Each human improves his performance to the goalbox until virtually all complete the trip in errorless and effortless fashion. At this point, CBS sets up a booth at the goalbox exit and Walter Cronkite interviews each human. "Could you tell me how you got here so quickly and so consistently?" Each human essentially replies, "I knew where the stocks and bonds were and I wanted them." This explanation would never be accepted, although one assumes that one can attribute human traits such as "knowing" and "wanting" to humans. It would not be acceptable because these words have to be translated into empirically acceptable terms. And it is this, not anthropomorphizing, that makes this "answer" unacceptable.

With respect to the "sin" of teleological explanation, while it makes sense in some cases to rule out explanations of a particular behavior based upon events that are subsequent to that behavior, a general rule to this effect is simply not logically sound. Obviously, one cannot say that a plate falls so that it can break or that an eye grows so that it may see. However, the possibility should be entertained that the reason these statements are unacceptable is that neither a plate nor an eye has an anatomical structure that can even be remotely construed as giving it control over its own behaviors. The longest observation of plates or eyes will not betray any self-initiated movements.

If, however, we have an organism that is capable of perceiving events and the relationship between events, is capable of storing or remembering these events and relationships, and has some degree of control over its own behavior, "teleological" hypotheses are no longer unacceptable, although they may be difficult to support empirically and, thus, may be unacceptable as explanations. This unacceptability does not, however, stem from the fact that the proposed explanation is "purposive," but from the difficulty of obtaining empirical support, a difficulty that, as noted above, might be just as great if the explanation were not purposive. The point is that a human has a nervous system that is capable of learning the relationship between two or more events and which is also capable of controlling a good part of its own behavior. Such an organism obviously can learn that a particular behavior is followed by a particular consequence, most of the time, or all of the time.

Thus, while a plate may not throw itself off a cliff in order to break-or because it wants to break-it seems quite possible that a human being might voluntarily emit a particular behavior, such as throwing himself off a cliff, if the fracturing that he perceived as an invariant consequence to his action was his ultimate goal. While one could counter that the causes of throwing oneself off a cliff precede the act, those causes would not generate the behavior if the probability of the perceived consequence were changed, by for example, installing a net a few feet below the edge of the cliff.

What we have been doing in many cases is eliminating suggested answers (and perhaps many of those answers should be eliminated) for reasons that are probably in error. These answers are not unacceptable because they are teleological, or because they reflect anthropomorphizing. They are unacceptable because, at the present time, they are not amenable to empirical test. Recognition of this could result in a confrontation with the true problem and perhaps in the development of a valid empirical test of such answers.

Psychology should be mature enough to recognize 


\section{4}

\section{BURSTEIN}

that it is dangerous to dismiss answers by labeling them. In my undergraduate days, students were advised that the question "What are the physical characteristics of the other side of the moon?" was empirically unanswerable. It is for this reason that answers such as "mind" and "green cheese" should be retained, rather than dismissed by labeling, not as explanations, but as reminders of the challenging type of problems that we face.

\section{REFERENCE}

Watson, J. B. Behaviorism. New York: Norton, 1925.

(Received for publication August 26, 1980.) 